\title{
Progress Report for the KAO 1.0 meter Robotic Telescope
}

Peter Mack ${ }^{1}$, Wonyong Han ${ }^{2}$, Matthew Bradstreet ${ }^{1}$, Anthony Borstad ${ }^{1}$, Jang-Hyun Park ${ }^{2}$, Ho Jin ${ }^{2}$, Woo-Baik Lee ${ }^{2}$ and Chung-Uk Lee ${ }^{2}$

${ }^{1}$ Astronomical Consultants \& Equipment, Inc., P.O. Box 91946, Tucson $A Z 85741$ USA

${ }^{2}$ Korea Astronomy Observatory, Taejon, 305-348, Korea

\begin{abstract}
Korea Astronomy Observatory (KAO) is working to rebuild a $1.0-\mathrm{m}$ robotic telescope in collaboration with a company (Astronomical Consultants \& Equipment, Inc. or ACE). The telescope is being totally refurbished to make a fully automatic telescope which can operate in both interactive an fully autonomous robotic modes. This paper describes the design concepts and the work completed. The telescope is an f/7.5 Ritchey-Chretien system mounted on an equatorial fork with friction drives capable of high slewing $\left(5^{\circ} / \mathrm{s}^{2}\right)$ and high resolution tracking. The control software manages the entire telescope, instruments and observatory. In interactive local and remote modes the observer can manually enter coordinates or retrieve them from a database. In robotic mode the telescope controller downloads requests from users and creates a schedule. The telescope will be equipped with a CCD camera and will be available over the internet.
\end{abstract}

\section{Introduction}

In order for small telescopes to compete with the very largest telescopes they have to be optimized for maximum efficiency, both in terms of optical throughput and the manner in which they are used. A fully autonomous robotic telescope (one that requires no human intervention) can be used to perform repetitive tasks and take advantage of only a few hours of partially clear nights which would not be utilized by conventional observing techniques. The rapid pre-scheduled observing sequence permits many more observations per night than could possibly be hoped for using the telescope manually with a well experienced observer. We present the design concepts for a meter-class telescope and observatory control system that permits interactive, remote and fully autonomous observing. The telescope is equipped with a finder-guider system and a CCD imaging detector with dual filter wheels. A pre-existing telescope was gutted to salvage the optics and servo motors for use in a new system.

\section{Control System Logistics}

A block diagram of the ACE Robotic Control System ${ }^{T M}$ is presented in Figure 1. The system is normally operated by three computers. The web computer 


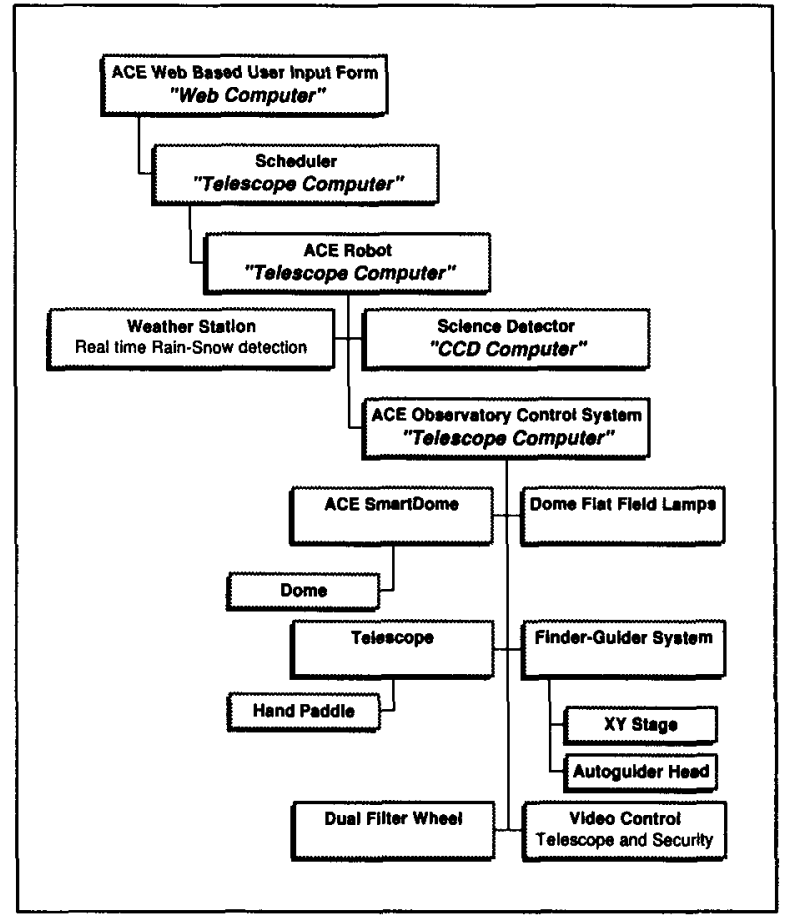

Figure 1. ACE Robotic Control System ${ }^{T M}$ logistics

is usually physically located at the administrative headquarters. It acts as a server for users to $\log$ on and request observations using an internet browser. The telescope computer and instrument computer handle the hardware at the observatory using a fast local area network.

\subsection{Web Computer}

Users request observations by logging on to a series of web pages. The address of this computer is well published and for security reasons it is not located at the observatory. There are three levels of user (Table 1) designed for "public", "student" and "professor". Public access is restricted to simple requests. Privileged users can request multiple sets of observations (the whole sequence to be repeated at some later time) with mosaics (multiple fields), multiple filters, and multiple exposures per filter. In order to stop any one observer from grabbing the entire resources of the telescope a priority scheme has been devised. The system administrator allocates a certain number of points or credits to each observer. Points are spent according to a simple formula:

$$
\text { Points used }=\text { Priority Level } \times \text { observation time. }
$$

A given level of observer has a range of Priority Levels. Objects requiring many repeat observations might be given a low priority level and those requiring one observation that is vital would be given the highest possible priority level. 
Table 1. User Priorities

\begin{tabular}{lccccc}
\hline User & $\begin{array}{c}\text { Priority } \\
\text { Level }\end{array}$ & $\begin{array}{c}\text { Repeat Sets of } \\
\text { Observations }\end{array}$ & $\begin{array}{c}\text { Multiple } \\
\text { Filter }\end{array}$ & $\begin{array}{c}\text { Multiple Exposures } \\
\text { per Filter }\end{array}$ & $\begin{array}{c}\text { Mosaic } \\
\text { Fields }\end{array}$ \\
\hline Public & 1 & No & Yes & No & No \\
Student & $2-4$ & Yes & Yes & Yes & Yes \\
Professor & $5-7$ & Yes & Yes & Yes & Yes \\
Transient & 8 & No & Yes & Yes & No \\
Engineer & 9 & Yes & Yes & Yes & Yes \\
\hline
\end{tabular}

There is also a Priority Level for transient events, such as gamma-ray bursts. When a "Level 8" event is received by the system the current observation is halted and the telescope is immediately pointed to the transient event unless an "Engineer" is logged on the system for maintenance work or another Level 8 observation is in progress.

The web pages support solar system observations, such as planets, asteroids and comets. This takes a different route through the pages because the position of the object depends on the time it will be observed. Positions are therefore generated in real time by the telescope computer. For comets and asteroids orbital elements are required.

The web pages also have links to the major astronomical databases. After successful submission of an observing request confirming e-mail is sent to the user. After the observation has been completed the images are achieved on the web computer and further e-mail is sent to the user with retrieval instructions. The address of the telescope and CCD computer are never revealed to users accessing the web pages.

The resolution and format of this paper does not allow us to present sample web pages. Readers are encouraged to inspect the pages by visiting http://www. astronomical.com and following the appropriate links.

Successful completion of the web pages generates an entry in the request database. The requests are also stored in RTML (Robotic Telescope Markup Language) format. The ACE Robotic Control System ${ }^{T M}$ was the first control system to implement and operate a telescope using RTML. The KAO Telescope will be capable of participating in an array of networked telescopes.

\subsection{Telescope and Camera Computers}

The telescope computer is located in the observatory control room. It receives requests from the web computer. It is also possible for a privileged observer to directly $\log$ on to this computer and operate the telescope either from the observatory or remotely using remote access software.

The telescope computer manages the ACE Robot. This generates a schedule, and makes the observations by communicating with the telescope controller and the CCD camera(s) using a series of NT pipes. The ACE software was written using Visual $\mathrm{C}++$ to create native 32-bit applications executing under Microsoft Windows NT. The computer is a dual Pentium motherboard housed in an industrial rack-mount chassis. To maximize the number of interrupts avail- 
able for instrument control the computer has dual-channel SCSI built into the motherboard. This requires less interrupts than IDE drives, is faster, and easily expandable. The telescope computer is equipped with two motion control cards, each having four axes of control with encoder feedback, a 24 channel digital input-output card, a GPS-based clock card for precision time keeping, and a video capture card for placing real-time video on the computer screen and on the internet. The video capture card uses a PCI slot, so there is just one PCI slot remaining for future expansion. A separate computer manages the scientific detector, usually a charged coupled device (CCD) camera. This is necessary because of computing loads during CCD readouts, and the lack of available slots in the telescope computer.

\subsection{ACE Control Crate}

The ACE Control Crate is the interface between the computer and the instrumentation. It too is rack-mounted and three cables for the motion control cards and the digital I/O card provide communication between the crate and the computer. The ACE Control Crate contains power supplies and optically isolated relays (input and output) to protect the computer from spurious electrical spikes. All the control cables from the telescope, dome, finder-guider, filter wheel and video control are terminated at this crate.

\subsection{ACE Smart Dome D $^{T M}$}

The observatory dome is automatically slaved to the telescope. One of the most important aspects of remote and robotic observing is to ensure that the dome can always be closed. An embedded microprocessor, the ACE SmartDome ${ }^{T M}$, constantly handshakes with the computer. In the event of a computer crash the device automatically sends the dome home and closes the shutter(s). The device has an RS232 communications port to permit full dome status information to be sent to the control computer.

\subsection{Video Subsystem}

The video subsystem uses a commercial color PAL/NTSC video switcher to permit one of eight cameras to be viewed, or a quad display of the first or last four cameras. The view can be switched in software by sending a simple binary pattern to the switcher using the digital I/O card. The output from the video switcher is also fed into an inexpensive video capture card to permit real-time video to be placed on the internet. The exact arrangement of the cameras is to be determined. Typical uses will be a color cloud camera, a low-light level camera for viewing the telescope at night, a dome camera to view the dome slit, a low light level color camera equipped with a $200 \mathrm{~mm}$ lens to view the Sun or Moon during eclipses for public outreach (broadcast live on the internet), and some security cameras. The system is also equipped with relays to toggle low-wattage lights inside the dome for viewing the telescope at night.

\subsection{Finder-Guider System}

The finder-guider system uses a pick-off mirror to gain off-axis access to three sides of the CCD field. It is held on sliding tables using crossed roller bearings 
and driven with micro-stepped motors. Internal optics image the focal plane and send parallel light along a compensating arm before being re-imaged by another lens to produce a 2 arc minute field of view at $2.3 \mathrm{X}$ magnification of the telescope. An Apogee instruments MegaLisaa CCD camera will be used for autoguiding.

\subsection{Filter Wheel}

An ACE Dual Filter Wheel is attached to the underside of the finder-guider box. This has two stacked filters wheels, each with ten filter slots to accommodate 3 inch square filters. One slot in each wheel is left open giving a total capacity of 18 filters. It is equipped with an absolute encoder and is driven by stepper motors. One advantage of the dual filter wheel is that neutral density filters can be placed in the upper wheel to permit multicolor photometry of very bright objects using the lower wheel. The filter wheel can take a CCD dewar up to $200 \mathrm{~mm}$ diameter with a weight of $20 \mathrm{~kg}$.

\subsection{GPS Clock}

A global positioning satellite (GPS) clock is used to keep the telescope computer clock synchronized to better than 0.05 seconds. It checks once a minute and resets the clock if there has been a drift. The CCD computer is also reset using a Windows-NT time service. The telescope control software calculates sidereal time knowing the universal time from the GPS card.

\section{The Telescope}

\subsection{The Optics Design}

The primary mirror was taken from a pre-existing telescope and is a lightweight $(70 \mathrm{~kg})$ honeycomb substrate manufactured by HexTek Corporation of Tucson with a f/2.63 focal ratio. The front and back surfaces are approximately 11 $\mathrm{mm}$ thick and both are curved such that in cross section they are parallel to each other. The focal ratio of the primary could not be significantly altered. It was decided to open up the size of the central hole and to manufacture a new secondary so as to create a Ritchey-Chretien Cassegrain design with the widest possible field. The effective focal ratio is $\mathrm{f} / 7.5$, which gives an image scale of $26.25 \mathrm{arc}-\mathrm{seconds} / \mathrm{mm}$. and a useable field diameter of 28 arc minutes

Table 2 gives the optical design parameters. The available field of view of the telescope is limited by two factors: the size of the hole in the primary mirror/baffle system, and, to a lesser extent, the amount of acceptable residual astigmatism. The hole in the primary is only $150 \mathrm{~mm}$. The baffle system was designed to act as both the sky flood baffle and the mirror handling fixture. The wall thickness of the tube has been minimized to allow the largest possible light cone to enter. However, a typical R-C Cassegrain might have a central hole of approximately $225-250 \mathrm{~mm}$ diameter. The honeycomb design of the mirror prevents us from opening up the size of the central hole by more than a few millimeters. However, we have gone ahead and done this to help insert the baffles. 
Table 2. Optical design parameters for KAO $1 \mathrm{~m}$ telescope

\begin{tabular}{lll}
\hline System Component & Properties & Value \\
\hline Primary Mirror & Diameter (physical) & 1046 \\
& Diameter (optical) & 1046 with 1000 test area \\
& Radius of curvature & 5508.9 \\
& Focal Ratio & 2.633 \\
& Conic Constant & -1.1092 (weak hyperbola) \\
Secondary Mirror & Diameter (physical) & 332 \\
& Diameter (optical) & 330.4 \\
& Radius of curvature & -2606.7 \\
System Characteristics & Conic Constant & -5.6389 \\
& Effective Focal Length & 7845 \\
& Effective focal ratio & $\mathrm{f} / 7.5$ \\
& Field of view (diameter) & 0.2334 (840 arc seconds) \\
& Plate Scale & 26.25 arcseconds/mm \\
Spacings & Prime focus intercept & 845.7 \\
& Primary-Secondary spacing & 1908.7 \\
& Focus ram travel & 50 \\
& Back focal distance & 500 \\
\hline
\end{tabular}

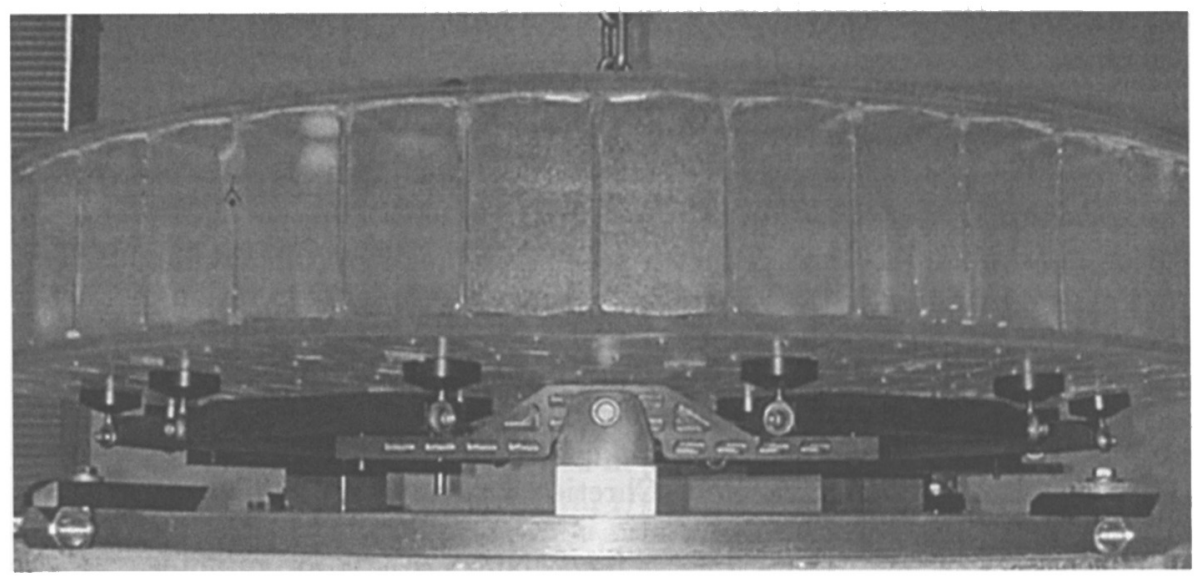

Figure 2. The mirror support system is used when the mirror is repolished.

\subsection{Optical Tube Assembly and Mounting}

The telescope mount is an equatorial fork, designed for rapid movement during long slew motions across the sky. It uses friction drives rather than the traditional worm drives to minimize backlash. The optical assembly will have an open truss design for optimum seeing performance and minimal mechanical and thermal inertia. 


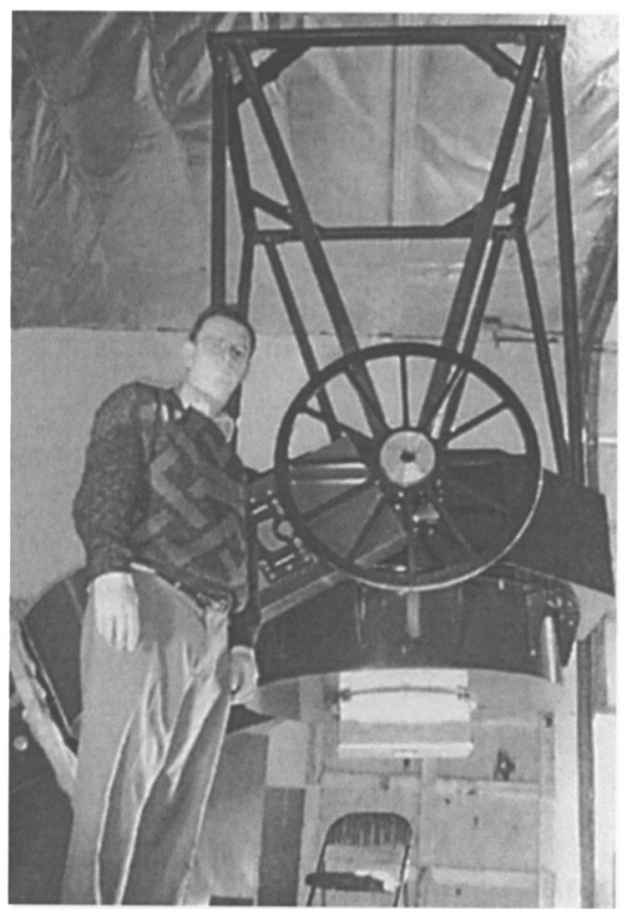

Figure 3. The telescope during assembly in December, 2000.

The mirror cell supports the mirror on an 18-point elastomeric mounting (Figure 2). It was engineered to provide the optimum support and not to hide any of the mirror chambers which would cause damage when the glass is placed in the aluminizing tank. The support system has been tested by tilting the mirror below the horizon for many days. The support system was also attached to the optical polishing machine so the optical figure was generated and tested as if the mirror were in the telescope. The optical tube assembly is equipped with automatic covers. The top end of the optical assembly is fixed so that only one secondary mirror is used. The secondary is supported using a four-vane spider. The focus ram has a precision ball-screw that drives a crossed-roller slide that is driven by a stepper motor with closed-loop encoder feedback. The control system permits re-initialization of the encoder to the OUT position. Current focus values are retained, even during complete power-down.

The Hour Angle and declination axes are both friction disk drives (Figure 3) employing Compumotor Dynaserve servo motors. These motors have sufficient static torque that they can hold the telescope even with the power off, assuming the telescope is in reasonable balance (within $5 \mathrm{Nm}$ ). If the telescope is purposefully placed out of balance, such as when changing instrumentation, a set of braces is used to keep the telescope tube pointing at the zenith. The telescope has a mercury tilt switch horizon sensor, which is set to 10 degrees. In additional there are sensors to prevent polar wrap and interference on the southern horizon 
with the fork arms. A home sensor permits automatic re-initialization of the telescope in the event of a computer malfunction. The disk to roller drive ratio is 53.3:1 for the hour angle axis, resulting in discrete steps on the sky of 0.05 arc seconds and a theoretical maximum slew rate of 13.5 degrees/second.

\section{Summary}

The KAO robotic telescope has been designed for efficient autonomous observing. It will be placed in active service in 2001 using a new mount and control system and refurbished optics. 